\title{
QoS Analysis to Optimize the Indoor Network IEEE 802.11 at UNTELS
}

\author{
Jhon S. Acuña-Aroni ${ }^{1}$, Brayan W. Alvarado-Gomez ${ }^{2}$, Avid Roman-Gonzalez ${ }^{3}$ \\ Department of Electronics and Telecommunications Engineering ${ }^{1,2}$ \\ Aerospace Sciences and Health Research Laboratory $(\text { INCAS-Lab })^{3}$ \\ Universidad Nacional Tecnológica de Lima Sur, Lima, Peru ${ }^{1,2,3}$
}

\begin{abstract}
This paper arose from the need to improve mobility and connectivity to network users of the Universidad Nacional Tecnológica de Lima Sur and the problems that arise on the quality of services $(\mathrm{QoS})$ such as signal intermittence, high latency, low decibels of received power, low coverage equipment that doesn't support high data transit and exceed the limit of connected users. An analysis is presented for optimization of the IEEE 802.11 wireless QoS in indoor media, the coverage data collected from the different equipment based on reception power measurements made with the Wi-Fi Network Analyzer software, mapping of the ideal approach to new coverage areas and the location of Access Points in areas with high student, administrative and teaching staff of the university through simulations of the coverage of the equipment made with NETSPOT software. The results obtained show that the actual design of the wireless network presents interference by equipment configured in the same transmission channel and adjacent, as well as insufficient coverage of radiated power for indoor environments due to the poor location and choice of models of access points, and to be replaced with Access point with high density characteristics of users connected simultaneously by equipment, suitable for indoor environments with technologies such as MU-MIMO and ARM; the results are shown favorable and optimized.
\end{abstract}

Keywords-Access point; QoS; coverage; indoor; software

\section{INTRODUCTION}

Wireless networks are growing rapidly and rapidly due to its great advantage of installation, flexibility and scalability, being the most used 802.11 standard known worldwide by its trade name as Wi-Fi so-called by Interbrand who was hired by the founding members of the Wireless Ethernet Compatibility Alliance [1], now known as Wi-Fi Alliance, the organization that promotes Wi-Fi technology and created a standard of connection with multiple devices that certified the interoperability of equipment under the protocol IEEE 802.11 [2].

The use of this type of network is directly linked to the number of Internet users whose rate of growth influences proportionally. In 2018 the Internet had a number of users of 4,021 million, by contrast, 2019 has to date 4,388 million Internet users, $57 \%$ of the world population, representing an increase of $9.1 \%$ in 1 year. Also, $52 \%$ of Internet users have connected via wireless [3], a figure that shows that the problems that can arise in this type of network configuration would involve more than half of users in the world.
Many problems arise when implementing a network, greater is the case when it is a wireless network, this due to the hostile environment as gauging, temperature, distance and all kinds of natural barriers that faces the signal during the Transmitter/Receiver journey being impossible from the beginning to satisfy the QoS in its entirety and for all cases. [4].

It is becoming more and more difficult to ignore when problems arise to a large extent as a result of not having an adequate design. According to studies carried out in environments with an adequate indoor design (buildings), the best results considering the normalized throughput as an efficiency measure are obtained with a network based on the IEEE $802.11 \mathrm{n}$ standard with $78 \%$, while with IEEE $802.11 \mathrm{~b}$ an efficiency of $17 \%$ [5] is obtained, being a very low percentage of signal use even in the best of scenarios (78\%).

However, when a cable installation system cannot be used because the environments and conditions do not allow it, Wi-Fi becomes a relevant object of study. In the case of a university where students can access the network more easily From mobile devices, Wi-Fi cannot be ignored despite the many adverse factors that arise.

The present work the objective is to analyze the functioning of the network in order to propose solutions in the parameters of QoS to optimize the use of an indoor Wi-Fi network analyzing the delays, loose packages, jitter and badly routed packages that can damage the data transmission [6], in the case of the Universidad Nacional Tecnologica de Lima Sur (UNTELS the primary purpose is to provide users with a quality of service that meets the minimum standards and highlight the importance of the work in front of current technological needs where a good network connection means to provide better results in academic and work activities where a good network connection means to provide better results in academic and academic activities.

In the related works we can find the publication of the researcher R. Costa in May 2019 [7], where he analyzes how to control in real-time the communications under the same 802.11 standard but in the industrial field where there are essential problems such as those proportional to the installation environment, or the work of Ljiljana Simić in 2017 [8], where he studied the impact of transmission channels to improve performance in high-density indoor networks, and demonstrated that $80 \mathrm{MHz}$ full channels are beneficial only in dense networks with loads of Extremely high traffic. 
In the following section, the methodology used, the propagation model in indoor systems, and the main equations for the correct analysis of service quality will be known. Likewise, the b / $\mathrm{n}$ configurations of the IEEE 802.11 standard protocol and a quick explanation of the use of the software used, so that each user can apply them at the time of locating the access points and obtain the most efficient possible resource of each equipment. Section III will analyze the results obtained theoretically and those obtained in real-time with the software, considering a new design in case the parameters in $\mathrm{dBm}$ are not within the ranges of a good signal.

\section{Methodology}

For the analysis of the Wi-Fi network must take into account the correct order of data collection and interpretation as a fundamental part of the study, for this a flow of necessary actions must be taken into account.

"Fig. 1" shows the methodology used to analyze whether the actual Wireless QoS network or a new drafting system is better as a starting point, the battery status is the 802.11 standard system and the ITU-R P.1238-9 standard, if the network meets the requirements to obtain a data collection with the software for the power of the Wi-Fi signal, the last step to analyze the results and the verification with the propagation model inside ITU - R P.1238- 9. According to the results, it is possible to determine if it is necessary to apply a new design to optimize the Wireless QoS in the network.

\section{A. Model of Indoor Propagation}

Indoor propagation is based on the model of ITU-R Recommendation P.1238-9, updated in 2017, and provides propagation data and prediction method of high importance for the design of indoor and local area radio communication systems in the frequency range $300 \mathrm{MHz}$ to $100 \mathrm{GHz}$ [9].

The basic model is as follows, Eq. 1:

Ltotal $=20 \log f+N \log d+L f(n)-28$

Where:

$-N$ : power loss coefficient. See Table I.

$-f:$ frequency $(\mathrm{MHz})$

$-d$ : separation distance $(\mathrm{m})$ between the transmitting equipment and the receiving terminal (where $\mathrm{d}>1 \mathrm{~m}$ )

$-L f$ : soil penetration loss factor $(\mathrm{dB})$. See Table II.

$-n$ : number of floors between the transmitter and the receiver terminal $(\mathrm{n} \geqslant 1)$.

To calculate the reception power, the Friis equation is proposed [10], Eq. 2:

$P r=P t+G t+G r-$ Ltotal

Where:

-Pr: receiving power $(\mathrm{dBm})$

-Pt: AP transmit power $(\mathrm{dBm})$.

$-G t$ : transmitter antenna gain $(\mathrm{dBi})$
$-G r$ : receiver antenna gain $(\mathrm{dBi})$

-Ltotal: radio path losses in media indoor $(\mathrm{dB})$

To find the reception power on mobile devices, the gain and transmission values of the Alcatel-Lucent OmniAccess Stellar AP1101 access point consulted in the datasheet must be taken into account, it is also necessary to measure the distance between both devices and Consider radio path losses.

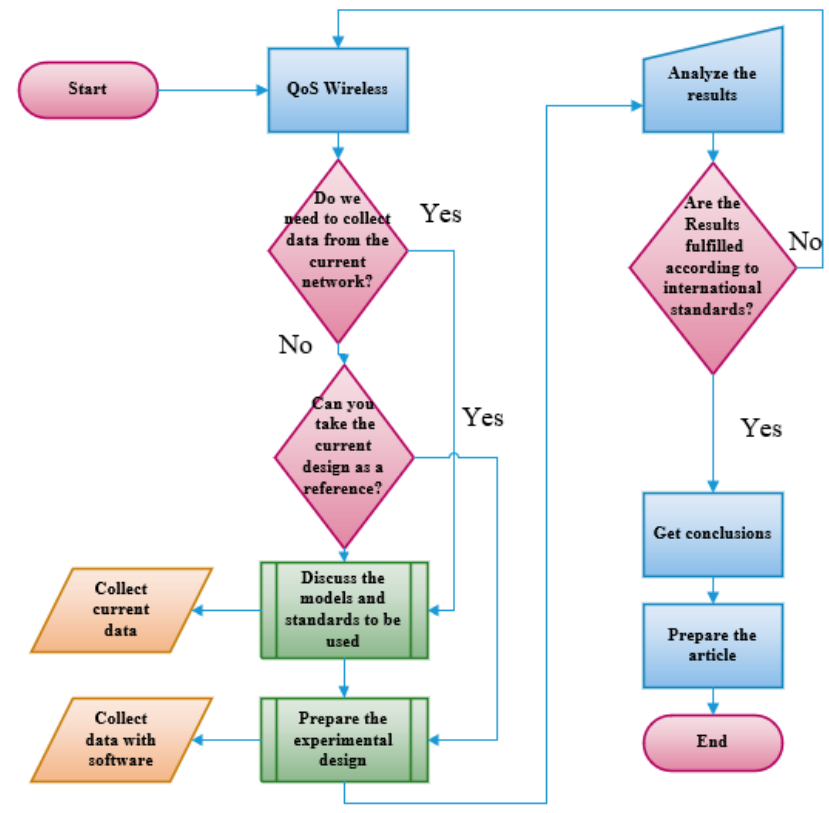

Fig. 1. Flow Diagram of the Methodology.

TABLE. I. COEFFICIENTS OF LOSS OF POWER, N, FOR THE CALCULATION OF THE LOSS OF TRANSMISSION IN INTERIORS. [9]

\begin{tabular}{|l|l|l|l|}
\hline Frequency & Residential building & Office building & $\begin{array}{l}\text { Commercial } \\
\text { building }\end{array}$ \\
\hline $900 \mathrm{MHz}$ & - & 33 & 20 \\
\hline $2.4 \mathrm{GHz}$ & 28 & 30 & - \\
\hline $5.2 \mathrm{GHz}$ & - & 31 & - \\
\hline $5.8 \mathrm{GHz}$ & - & 24 & - \\
\hline
\end{tabular}

TABLE. II. FACTORS OF LOSS OF PENETRATION IN THE SOIL, (DB), BEING N THE NUMBER OF FloORs PENETRATED, FOR THE CALCULATION OF THE LOSS OF TRANSMISSION INTERIORS $(\mathrm{N} \geqslant 1)$ [9]

\begin{tabular}{|l|l|l|l|}
\hline Frequency & $\begin{array}{l}\text { Residential } \\
\text { building(dB) }\end{array}$ & $\begin{array}{l}\text { Office } \\
\text { building }(\mathrm{dB})\end{array}$ & $\begin{array}{l}\text { Commercial } \\
\text { building }(\mathrm{dB})\end{array}$ \\
\hline $900 \mathrm{MHz}$ & - & $\begin{array}{l}9(1 \text { floors }) 19(2 \\
\text { floors }) 24(3 \\
\text { floors })\end{array}$ & 20 \\
\hline $2.4 \mathrm{GHz}$ & $\begin{array}{l}10 \text { (depart.) } \\
5 \text { (home) }\end{array}$ & 14 & - \\
\hline $5.2 \mathrm{GHz}$ & $\begin{array}{l}13 \text { (depart.) } \\
7 \text { (home) }\end{array}$ & $16(1$ floor $)$ & - \\
\hline $5.8 \mathrm{GHz}$ & - & $\begin{array}{l}22(1 \text { floor }) 28(2 \\
\text { floors })\end{array}$ & - \\
\hline
\end{tabular}




\section{B. Areas of Coverage}

The equipment that is currently implemented should be analyzed to study its characteristics after reviewing its corresponding datasheet and making a comparison with other equipment of similar or better characteristics that suits the purpose according to capacity and location. To make this discovery of equipment is necessary to have software, in this case, the Wi-Fi Network Analyzer provides the list of access point used by the university, the devices have in their mac address with the following ID: 80:2a:a8, to discover the manufacturer must track the address with the online tool Mac Address Vendor Search or similar.

The manufacturer of the detected Access Point is Ubiquiti Networks Inc. of model Outdoor to $2.4 \mathrm{GHz}$ located in the external part of a pavilion to take advantage of the radiation of signal in interior and exterior environments.

Scanning the Access Point models also results in the number of AP's, a distribution of four devices located outside the pavilions.

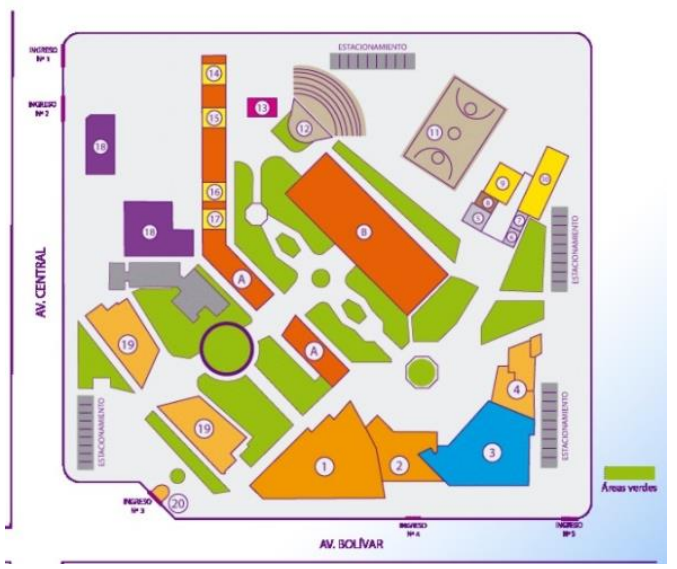

Fig. 2. Map of the Universidad Nacional Tecnológica de Lima Sur.

The indoor environments to study are:

A: Pavilion of halls of 1 row and 1 hallway

B: Pavilion of halls of 2 rows and 1 hallway

"Fig. 2" shows the map of the university campus indicating administrative offices, library, pavilions and other areas [11].

In-situ measurements of the pavilions provide data for the design of the structural plan in the Autocad 2020 program and to discover the coverage areas, the Wi-Fi Heatmaps software is used, previously loaded the imported plan and making the route through the interior environments connected to the wireless signal to be measured, in this case the Wi-Fi Untels network.

"Fig. 3" shows the heat map generated by the power in $\mathrm{dBm}$ of the Ap's and measured with the software in a terminal that has a wireless module of good sensitivity, drawing the map automatically as we move the terminal activated the GPS and varying the intensity of power received, wherein red the quality of signal received is bad, in yellow is regular and in green is good.

\section{Density of Users}

The study of the density of users is of vital importance when making the design of a Wireless network, according to recommendations raised the number of customers connected to an access point should be limited between 20 and 30 simultaneous users [12].

For carry out the analysis of the density of users, we proceeded to use the tool NetSpot or Wi-Fi Network Analyzer and vary the scanning periods in order to obtain results that show the number of users connected in a routine of the week.

These applications based on multithreaded union, use LAN scanner, in this scanner, every device connected to the local network is shown. Each device exports data such as hostname, IP address, MAC address, type of network security, among others. The software works by sending ARP (Address Resolution Protocol) messages to the broadcast belonging to the target network of the analysis.

\section{Problems during Connection}

The intermittence in the connection is product of the interference of signals, the biggest problem in the wireless networks, where the level of the problem is proportional in the difference of power of the signals radiated adjacently, that is to say, if two signals transmitted by the same channel, the transmitter that emits smaller power is the most affected by this phenomenon.
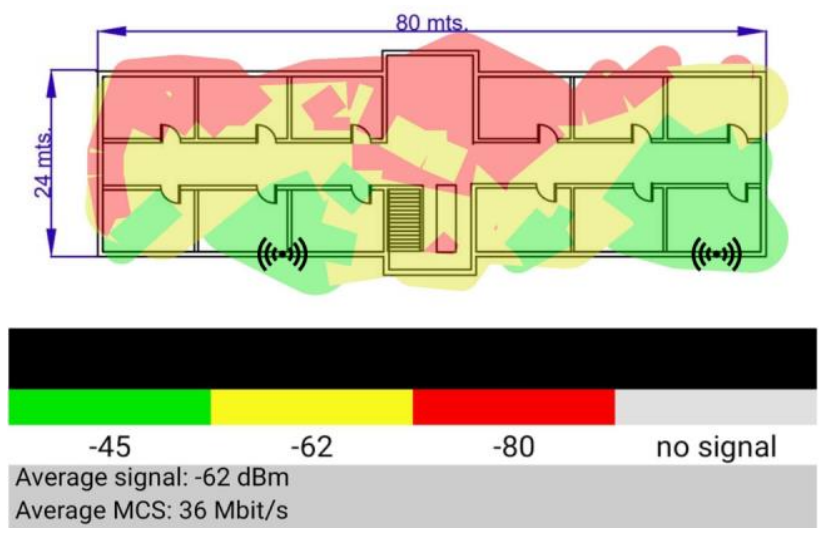

Fig. 3. Heat Map of Pavilion B.

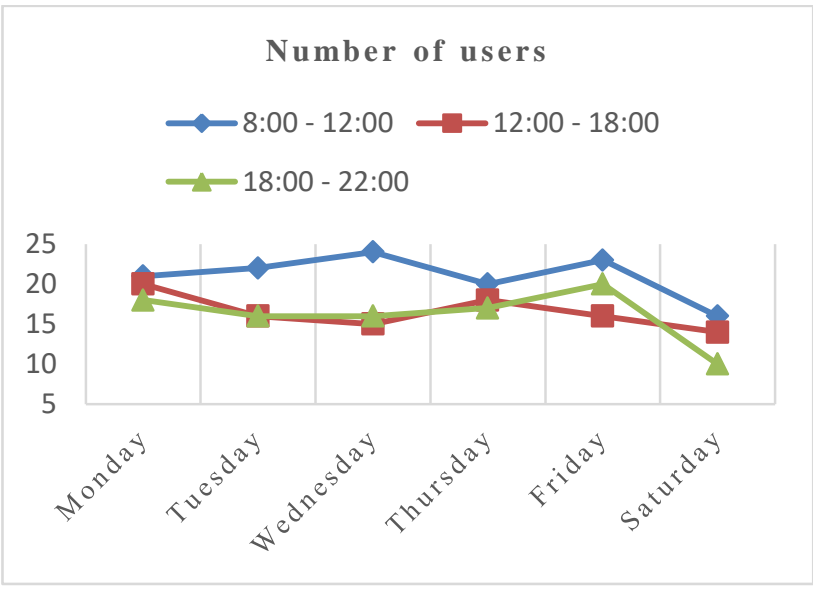

Fig. 4. The Density of users Connected to the Wi-Fi Untels Network. 


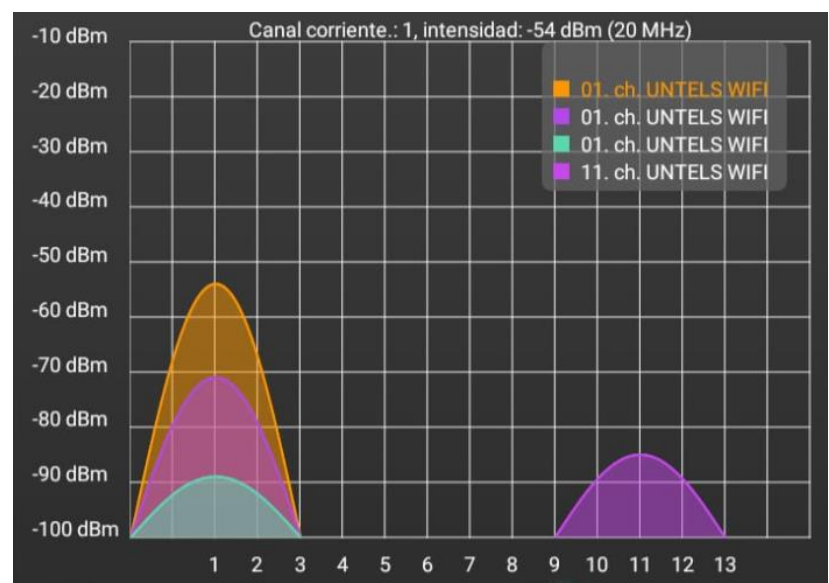

Fig. 5. Diagram of Transmission Channels with Wi-fi Network Analyzer.

With the use of the Wi-Fi Network Analyzer tool, we proceeded to scan the different radiated signals to find signals that are producing interference with each other and filter the results to observe the four access points of the objective of the analysis.

"Fig. 5" shows the transmission channels used with reference to radiated power where it is observed that work with channels 1 and 11 with a center frequency of $2.412 \mathrm{GHz}$ and $2.462 \mathrm{GHz}$, respectively.

\section{RESULTS}

To ensure a stable connection, the receiving power, must not be less than $-70 \mathrm{dBm}$. In the study carried out, see "Fig. 3", the radiated signal power of the two access points corresponding to pavilion B cannot cover the broad interior environment of 3 vertical floors, detecting zones where $70 \mathrm{dBm}$ are exceeded. Equipment should be replaced by indoor models and two access points placed in the center of each floor of the pavilions, as shown in "Fig. 6" to achieve adequate signal coverage.

The distribution for the new optimized design was done with 2 Alcatel-Lucent OmniAccess Stellar AP1101, for its ability to control the bandwidth assigned to each terminal dualfrequency transmission $2.4 \mathrm{GHz}$ and $5 \mathrm{GHz}$ and $2 \times 2 \mathrm{MIMO}$ technology, showing favorable results in "Fig. 7", reaching a signal quality higher than $-70 \mathrm{dBm}$ in almost all indoor environments. The floor structure is homogeneous; therefore, the design will be repeated in each floor and also for pavilion A, which has a smaller area dimension.

The density of connected users in "Fig. 4" reflects a positive comparison with the recommendations referenced in [10], ruling out equipment saturation.

The access points can be configured in such a way that one can automatically choose the channels to work. In the analysis, it was possible to find overlapping frequencies that generate interference and transmission delays between three equipments configured in channel 1; only the fourth access point works optimally on channel 11. This problem can be corrected by changing the configuration of the equipment in different channels, AP1 in channel 1, AP2 in channel 4, AP3 in channel 8 and AP4 in channel 11 or whether to activate the Adaptive Radio Management (ARM) option if the equipment has this technology.

The heat map obtained by software does not offer an error of $0 \%$, in order to obtain a more accurate study; it is compared with the results obtained from the equations presented in the previous section, with values of the measurements made onsite as represented in "Fig. 8".

Table III shows the losses of the radio path in indoor media, these losses depend directly on the places where the equipment will be installed with respect to the structure of the building.

The results obtained from Eq. 1 and 2, at a frequency of 2.4 $\mathrm{GHz}$ based on the ITU-R P.1238-9 standard; result in a minimum error of $8.7 \%$ and a maximum error of $9.67 \%$ with respect to the measured values.

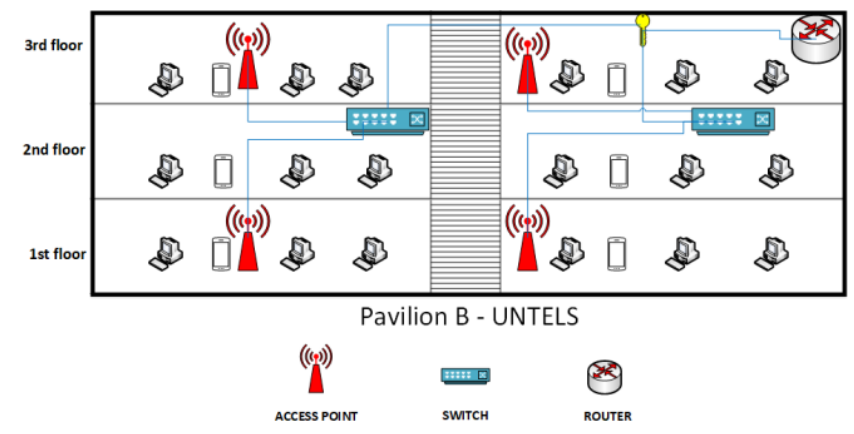

Fig. 6. The Ideal Location of Indoor Equipment.
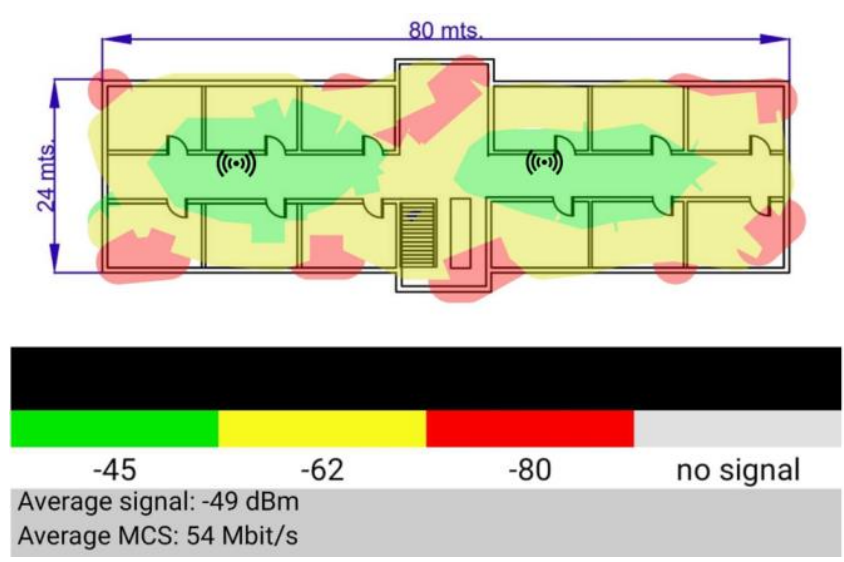

Fig. 7. Diagram of Transmission Channels with Wi-Fi Network Analyzer.

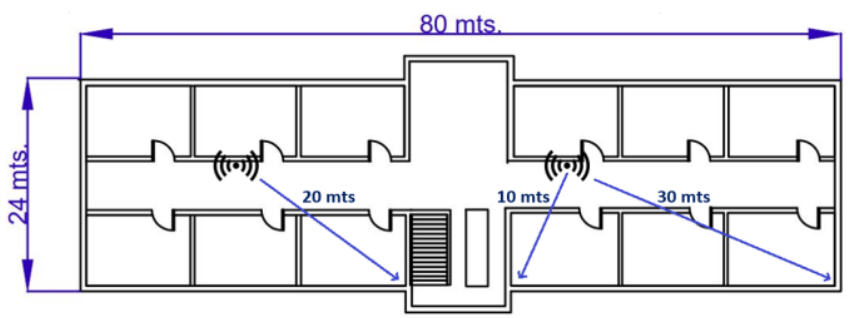

Fig. 8. Distances between the Receiver and Transmitter Equipment. 
TABLE. III. Results of TheORETICAL POWER AND MEASUREMENT

\begin{tabular}{|c|c|c|c|}
\hline \multicolumn{4}{|c|}{ Results with Stellar AP1101 } \\
\hline \multicolumn{4}{|c|}{ Loss of radio path (Ltotal): } \\
\hline \multirow{2}{*}{$\begin{array}{l}\text { ITU-R } \\
\text { P.1238-9 }\end{array}$} & 10 meters & 20 meters & 30 meters \\
\hline & $-83.60 \mathrm{~dB}$ & $-92.64 \mathrm{~dB}$ & $-97.92 \mathrm{~dB}$ \\
\hline \multicolumn{4}{|c|}{ Receiving Power (Pr): } \\
\hline \multirow{2}{*}{ Friis, Eq. 2} & 10 meters & 25 meters & 30 meters \\
\hline & $-63.2 \mathrm{dBm}$ & $-72.24 \mathrm{dBm}$ & $-77.52 \mathrm{dBm}$ \\
\hline \multirow{2}{*}{$\begin{array}{l}\text { Measured } \\
\text { value }\end{array}$} & 10 meters & 25 meters & 30 meters \\
\hline & $-57.09 \mathrm{dBm}$ & $-65.96 \mathrm{dBm}$ & $-70.01 \mathrm{dBm}$ \\
\hline
\end{tabular}

\section{CONCLUSIONS}

Having an optimal quality of service in wireless networks requires good design based on standards and measurements as a fundamental basis for implementation.

The transmission channels when working at $2.4 \mathrm{GHz}$ have very few transmission channels, specifically 14 , which is why interference is a complicated factor to correct as discussed in this paper. Currently, the most recent equipment has $5 \mathrm{GHz}$ technology that has 25 transmission channels not overlapped and under the same standard 802.11.

The distribution and choice of transmitting equipment must be designed according to the field of application, another important factor is the capacity, the configuration and design must be done always leaving availability to scale based on the results obtained.

However, this study presents some limitations such as the number of equipment tested, in this case, according to the results, we opted for an equipment whose characteristics theoretically optimized the QoS. Also, the access to data and information of configuration of the main router of the network was limited by the Office of Information Technologies and Communication of the university. Due to administrative reasons and to safeguard the confidentiality of information that circulates through the VLANs of management. For a more accurate study, these limitations can be remedied with greater investment and try with equipment of different models and features that of a better cost-benefit.
For future research it is advisable to analyze and better understand the phenomenon of refraction, reflection, and diffraction in the radio waves produced by wireless routers, it is also necessary to obtain the power losses caused by the type of material in the walls and even the 3D design of the infrastructure using more specialized software, it is also important to consider the environmental conditions that cause losses in signal power. The software used under Recommendation ITU-R P.1238-9 resulted in a minimum error rate of $8.7 \%$, for better results it is recommended to work with the software of greater sensitivity and precision.

\section{REFERENCES}

[1] TAGUAS, J. L. C. Navegando sin cables: Wi-Fi., 15 de febrero de 2009, pp. 4-5.

[2] Herrera-Ramírez, E., Díaz-Ramírez, A., \& Calafate, C. T. (2007). Desarrollando el estándar IEEE 802.11 n, un paso adelante en WLAN. In Segundo Congreso Internacional en Ciencias Computacionales, Baja California, México.

[3] Kemp, S. (2019). Digital 2019: Global Internet Use Accelerates. Retrieved from we are social.

[4] Ubom, E. A., Akpanobong, A. C., \& Abraham, I. I. (2019). Characterization of Indoor Propagation Properties and Performance Evaluation for 2.4 GHz Band Wi-Fi. Available at SSRN 3391700.

[5] Cueva, R. L., Jimenez, C. F., \& Maldonado, C. M. (2016). Análisis del desempeño en un enlace descendente de redes basadas en los estándares IEEE 802.11 b IEEE 802.11 ny WDS. Reci, 5(10).

[6] Felipe, C., del Rosario, M., Martínez Gómez, R., \& Crespo García, Y. (2013). Análisis de la QoS en redes inalámbricas. Revista Cubana de Ciencias Informáticas, 7(1), pp. 86-96.

[7] R. Costa, J. Lau, P. Portugal, F. Vasques, and R. Moraes, "Handling real-time communication in infrastructured IEEE 802.11 wireless networks: The RT-WiFi approach," in Journal of Communications and Networks, vol. PP, no. 99, pp. 1-15, May 2019.

[8] Simić, L., Riihijärvi, J., \& Mähönen, P. (2017, June). Measurement study of IEEE 802.11 ac Wi-Fi performance in high density indoor deployments: Are wider channels always better?. In 2017 IEEE 18th International Symposium on A World of Wireless, Mobile and Multimedia Networks (WoWMoM). IEEE.

[9] Series, P. (2017). Propagation data and prediction methods for the planning of indoor radiocommunication systems and radio local area networks in the frequency range $300 \mathrm{MHz}$ to $100 \mathrm{GHz}$. Recommendation ITU-R, 1238-9.

[10] Levis, C., Johnson, J. T., \& Teixeira, F. L. (2010). Radiowave propagation: physics and applications, Chapter 5. John Wiley \& Sons.

[11] Universidad Nacional Tecológica de Lima Sur (2019). Mapa Campus Universitario. [plane].

[12] Florwick, J., Whiteaker, J., Amrod, A. C., \& Woodhams, J. (2013). Wireless LAN design guide for high density client environments in higher education. Cisco Systems Design Guide, pp. 5-6. 